\title{
BÄCKLUND TRANSFORMATIONS FOR SEVERAL CASES OF A TYPE OF GENERALIZED KdV EQUATION
}

\author{
PAUL BRACKEN
}

Received 4 August 2004

\begin{abstract}
An alternate generalized Korteweg-de Vries system is studied here. A procedure for generating solutions is given. A theorem is presented, which is subsequently applied to this equation to obtain a type of Bäcklund transformation for several specific cases of the power of the derivative term appearing in the equation. In the process, several interesting, new, ordinary, differential equations are generated and studied.
\end{abstract}

2000 Mathematics Subject Classification: 35A15, 35C05, 35A25.

There has been considerable interest recently in the study of generalized nonlinear Korteweg-de Vries (KdV) equations. One in particular has been investigated in a number of papers $[4,5,8]$, and recently many explicit solutions to the equation have been produced [1]. The exact form of this equation is given as

$$
w_{t}+a\left(w^{p}\right)_{x}+b\left(w^{q}\right)_{x x x}=0
$$

where $a$ and $b$ are real, nonzero constants. When $p=q=1$, the equation becomes a linear equation, and when $p=2$ and $q=1$, the classical $\mathrm{KdV}$ equation results [2]. The symmetry group has been determined and group invariant solutions have been produced as well [1]. The interest in this type of equation resides in the fact that nonlinear dispersion is taken into account, and moreover, it has been realized that nonlinear dispersion can act to compactify solitary waves and generate solitons which have a finite wavelength. This new type of soliton has been referred to as a compacton, and it can be thought of as a soliton which has a finite wavelength. It is likely that this type of equation will find many applications in the areas of condensed matter physics and statistical mechanics.

It is the intent here to investigate a related set of equations which are of interest and given in the form

$$
w_{t}+w_{x x x}+g^{\prime}\left(w_{x}\right)=0
$$

where $g(t)$ is a differentiable function of a single variable, in this case, a polynomial. Equation (2) can be thought of as a type of extension of the KdV equation when $g$ is selected appropriately. The equation can also manifest nonlinear dispersion depending on the form of $g$ as in the equation above. The intention here is to show that a class of solutions for (2) can be generated by integration. Then, it will be shown that for a particular form of the function $g$, a type of Bäcklund transformation can be obtained. 
This is still a relatively new subject now, and what will be presented here is meant to initiate further work.

Consider a pair of partial differential equations, which can be expressed in the form

$$
\Delta(u)=0, \quad \Sigma(v)=0,
$$

where $u, v$ denote the functions to be determined, while $\Delta$ and $\Sigma$ represent particular differential operators in $m$ independent variables. Here, the case in which there are two independent variables which will be referred to as $t$ and $x$ will be treated. A Bäcklund transformation would constitute a system of relations which involve $u, v$, and their corresponding derivatives such that they ensure that $v$ satisfies the second equation in (3) when $u$ satisfies the first, and conversely [3]. Suppose that the equation $\Delta(u)=0$ is the Euler-Lagrange equation of a variational principle corresponding to a particular Lagrangian $L(u)$. In addition, suppose there is a relation between $u$ and $v$ and their derivatives which imply that $L(v)-L(u)$ is a divergence. Then, $\Delta(v)=0$ holds whenever $\Delta(u)=0$ and conversely. From this, it follows that these relations would possess the Bäcklund property just defined for the pair of equations in (3). In this instance, (3) are referred to as a variational Bäcklund transformation [6, 7]. In the case of the generalized form (2), these are closely related to Euler-Lagrange equations, but are not identical. Let the Euler-Lagrange equations be written as $E_{j}(u)=0, j=1, \ldots, n$, which if the Lagrangian depends on second derivatives takes the form

$$
E_{j}(u)=\frac{d}{d x^{\alpha}}\left[\frac{\partial L}{\partial u_{\alpha}^{j}}-\frac{d}{d x^{\beta}}\left(\frac{\partial L}{\partial u_{\alpha \beta}^{j}}\right)\right]-\frac{\partial L}{\partial u^{j}},
$$

where $x^{\alpha}$ are the independent variables and $u^{j}$ the dependent variables and the subscript on $u^{j}$ indicates partial differentiation. However, another transformation, which will be referred to as a simple Bäcklund transformation, can be defined by requiring that it guarantees the difference $E(v)-E(u)$ vanishes. The idea here is to show that explicit solutions to (2) can be determined. Next, we give a simple but clear proof of a theorem which can be used to produce a simple Bäcklund transformation for equations of the form (2). For a particular choice of the function $g(z)$ in (2), namely,

$$
g(z)=\frac{\alpha}{n(n+1)} z^{n+1}
$$

it will be shown how to obtain specific transformations explicitly by applying Theorem 1 for several values of the power $n$ in (5). This method is of interest in itself, and in developing it, leads to other types of ordinary differential equations which must be integrated to produce the transformation and are themselves of interest. A straightforward transformation procedure is found, which leads to results, and it is hoped that this will stimulate further work in formulating generalizations of the procedure which is presented here.

To demonstrate the feasibility of generating at least some explicit solutions to (2), we look for a class of solutions to (2) of the form $w(x, t)=w(x-c t)$. Introduce the new variable $\xi=x-c t$ into (2) so that the derivatives with respect to $t$ and $x$ are replaced 
by derivatives with respect to $\xi$. In terms of the new variable $\xi$, (2) takes the form

$$
-c w_{\xi}+w_{\xi \xi \xi}+g^{\prime}\left(w_{\xi}\right)=0 .
$$

Set $\tau=w_{\xi}$ in (6) so that the order is reduced by one and it becomes

$$
-c \tau+T \xi \xi+g^{\prime}(\tau)=0 .
$$

Multiplying both sides of (7) by $T_{\xi}$, it can be written in the form

$$
-\frac{c}{2}\left(\tau^{2}\right)_{\xi}+\frac{1}{2}\left(\tau_{\xi}^{2}\right)_{\xi}+(g(\tau))_{\xi}=0 .
$$

Integrating (8) once, we obtain

$$
-c \tau^{2}+\tau_{\xi}^{2}+2 g(\tau)=C .
$$

Solving (9) for $\tau_{\xi}$, we obtain that $\tau_{\xi}^{2}=c \tau^{2}-2 g(\tau)+C$, hence (9) can be separated and written as a quadrature

$$
\int \frac{d \tau}{\sqrt{c \tau^{2}-2 g(\tau)+C}}=\epsilon \xi+K, \quad \epsilon= \pm 1 .
$$

Integrating on the left for particular $g$, we obtain something which depends on $\tau$. Solving this resulting expression, $\tau$ is obtained as a function of $\xi$ explicitly. This gives precisely $w_{\xi}$. Integrating a final time, we obtain $w(\xi)$. If $g$ is given in the form (5), then the quantity under the radical in (10) is a polynomial of degree $n+1$ in the variable $\tau$. In fact, the integral in (10) takes the form

$$
\int \frac{d \tau}{\sqrt{c \tau^{2}-(2 \alpha / n(n+1)) \tau^{n+1}+C}}=\epsilon \xi+K .
$$

Of course, (11) can be integrated easily in the case in which $c=0$ and $C=0$, but giving only static solutions. This can be integrated explicitly for certain values of $n$ and the constants to give nontrivial solutions as well. For example, suppose $n=3, \alpha=-6$, $c=2$, and $C=1$, then the solution to (2) is given by

$$
w(x, t)=\ln (\sec (x-2 t+K))+C_{1} .
$$

If we simply put $n=3$ and $C=0$ into (11), the following solution to (2) is obtained

$$
w(x, t)=2 \sqrt{\frac{6}{\alpha}} \arctan \left(\sqrt{\frac{2}{3} \alpha c} \exp (\sqrt{c}(x-c t+K))\right) .
$$

These solutions can be verified by direct substitution into (2). Elliptic function solutions could also be obtained from (11) as well.

Consider a general Lagrangian of the form

$$
L=\frac{1}{2} u_{t} u_{x}-\frac{1}{2} u_{x x}^{2}+g\left(u_{x}\right),
$$


where $u$ represents the dependent function. The Euler-Lagrange expression in this case reduces to

$$
\begin{aligned}
E(u) & =\frac{d}{d t}\left(\frac{\partial L}{\partial u_{x}}-\frac{d}{d x}\left(\frac{\partial L}{\partial u_{x x}}\right)\right)+\frac{d}{d t}\left(\frac{\partial L}{\partial u_{t}}\right) \\
& =u_{t x}+u_{x x x x}+\frac{d}{d x} g^{\prime}\left(u_{x}\right) \\
& =\frac{d}{d x}\left(u_{t}+u_{x x x}+g^{\prime}\left(u_{x}\right)\right)=\frac{d}{d x} \mathscr{E}(u) .
\end{aligned}
$$

A criterion for the existence of a simple Bäcklund transformation associated with the equation $\mathscr{E}(u)=0$ is given in the following theorem.

THEOREM 1. The partial differential equation (2) possesses a simple Bäcklund transformation relating $w$ and $z$, if for the given function $g\left(w_{x}\right)$, there exist functions $\varphi(v)$, $\theta(v)$ where $\theta^{\prime}(v) \neq 0$ such that the following condition is satisfied:

$$
g^{\prime}\left(z_{x}\right)-g^{\prime}\left(w_{x}\right)=-\frac{\theta^{\prime \prime \prime}(v)}{\theta^{\prime}(v)} v_{x}^{3}-2 \frac{\varphi^{\prime}(v)}{\theta^{\prime}(v)} v_{x}
$$

and $w$ and $z$ are related to $u$ and $v$ by the linear transformation

$$
w=u-v, \quad z=u+v .
$$

Under these conditions, the corresponding simple Bäcklund transformation has the form

$$
\begin{gathered}
u_{x}=\theta(v) \\
u_{t}=-\theta^{\prime}(v) v_{x x}+\frac{1}{2} \theta^{\prime \prime}(v) v_{x}^{2}+\varphi(v) .
\end{gathered}
$$

Proof. Setting $2 \chi=\mathscr{E}(z)-\mathscr{E}(w)$, where $\mathscr{E}(w)=w_{t}+w_{x x x}+g^{\prime}\left(w_{x}\right)$, we can write

$$
\begin{aligned}
2 x & =z_{t}+g^{\prime}\left(z_{x}\right)+z_{x x x}-w_{t}-g^{\prime}\left(w_{x}\right)-w_{x x x} \\
& =u_{t}+v_{t}-u_{t}+v_{t}+u_{x x x}+v_{x x x}-u_{x x x}+v_{x x x}+g^{\prime}\left(z_{x}\right)-g^{\prime}\left(w_{x}\right) \\
& =2\left(v_{t}+v_{x x x}\right)+g^{\prime}\left(z_{x}\right)-g^{\prime}\left(w_{x}\right) .
\end{aligned}
$$

Substituting (16) into (19), we obtain

$$
\begin{aligned}
\theta^{\prime}(v) \chi & =\theta^{\prime}(v) v_{x x x}-\frac{1}{2} \theta^{\prime \prime \prime}(v) v_{x}^{3}-\varphi^{\prime}(v) v_{x}+\theta^{\prime}(v) v_{t} \\
& =\frac{\partial}{\partial x}\left[\theta^{\prime}(v) v_{x x}-\frac{1}{2} \theta^{\prime \prime}(v) v_{x}^{2}-\varphi(v)\right]-\frac{\partial}{\partial t}[\theta(v)]
\end{aligned}
$$

By definition, the existence of a simple Bäcklund transformation which relates the functions $w$ and $z$ must imply that $\chi=0$. The result for $\theta^{\prime}(v) \chi$ in (20) then implies the existence of a function $U$ such that

$$
U_{x}=\theta(v), \quad U_{t}=-\theta^{\prime}(v) v_{x x}+\frac{1}{2} \theta^{\prime \prime}(v) v_{x}^{2}+\varphi(v)
$$


The identification of $u$ with $U$ immediately gives rise to the relations in (18), which specifies the transformation. Also, differentiating $u_{x}$ in (18) with respect to $t$ and $u_{t}$ with respect to $x$, the compatibility condition obtained by subtracting $u_{t x}$ and $u_{x t}$ is found to be

$$
u_{x t}-u_{t x}=\theta^{\prime}(v) v_{x x x}-\frac{1}{2} \theta^{\prime \prime \prime}(v) v_{x}^{3}-\varphi^{\prime}(v) v_{x}+\theta^{\prime}(v) v_{t}
$$

This vanishes precisely when $x=0$ from (20), and so the compatibility condition $u_{x t}=$ $u_{t x}$ holds.

The theorem can now be applied to obtain the coefficient functions $\theta(v)$ and $\varphi(v)$ which appear explicitly in the Bäcklund transformation (18). This can be done by solving a set of auxiliary differential equations for the case in which the function $g$ has the form (5). In this case, the differential equation takes the form

$$
w_{t}+w_{x x x}+\frac{\alpha}{n}\left(w_{x}\right)^{n}=0 .
$$

It will be shown that the cases $n=2,3$, and 4 can be treated in the context of the formalism described in Theorem 1 and presented in (18).

(1) Consider the case in which $n=2$ so that we can write

$$
g^{\prime}\left(z_{x}\right)-g^{\prime}\left(w_{x}\right)=\frac{\alpha}{2} z_{x}^{2}-\frac{\alpha}{2} w_{x}^{2}=2 \alpha u_{x} v_{x}
$$

This expression has exactly the form given by (16), however, there is no term proportional to $v_{x}^{3}$. To match (24) to this form, it suffices to suppose that $\theta^{\prime}(v)$ is not identically zero, since $v_{x}$ appears in (25), but require that $\theta^{\prime \prime \prime}(v)=0$ to prevent the appearance of the $v_{x}^{3}$ term. This forces the coefficient of $v_{x}^{3}$ to vanish, and implies that $\theta(v)$ is quadratic in $v$,

$$
\theta(v)=a+b v-\frac{\alpha}{6} v^{2}
$$

The coefficient of $v_{x}$ is then given by $2 \alpha u_{x}=2 \alpha \theta(v)$, and so $\varphi(v)$ is determined by the first-order equation

$$
-2 \frac{\varphi^{\prime}(v)}{\theta(v)}=2 u u_{x}
$$

Substituting for $u_{x}$ in terms of $\theta$, this is equivalent to

$$
\varphi^{\prime}(v)=-\alpha \theta(v) \theta^{\prime}(v)=-\frac{1}{2} \alpha\left(\theta(v)^{2}\right)^{\prime}
$$

Therefore, $\varphi(v)$ is obtained in the form

$$
\varphi(v)=-\frac{1}{2} \alpha \theta(v)^{2}
$$


The Bäcklund transformation which is specified by Theorem 1 can be written explicitly as

$$
\begin{gathered}
u_{x}=\theta(v)=a+b v-\frac{\alpha}{6} v^{2} \\
u_{t}=-\left(b-\frac{\alpha}{3} v\right) v_{x x}-\frac{\alpha}{6} v_{x}^{2}-\frac{\alpha}{2}\left(a+b v-\frac{\alpha}{6} v^{2}\right)^{2} .
\end{gathered}
$$

As an example, to show that the compatibility condition for (29) holds in this case, differentiate $u_{x}$ with respect to $t$ and $u_{t}$ with respect to $x$ to obtain the pair

$$
u_{x t}=b v_{t}-\frac{\alpha}{3} v v_{t}, \quad u_{t x}=-b v_{x x x}+\frac{\alpha}{3} v v_{x x x}-\alpha u_{x}\left(b v_{x}-\frac{\alpha}{3} v v_{x}\right) .
$$

Now, the defining constraint $\chi=0$ implies that $v_{t}=-v_{x x x}-\alpha u_{x} v_{x}$, which allows the elimination of $v_{t}$ from $u_{x t}$ giving

$$
u_{x t}=-b v_{x x x}-\alpha b u_{x} v_{x}+\frac{\alpha}{3} v v_{x x x}+\frac{\alpha^{2}}{3} u_{x} v v_{x}
$$

Subtracting $u_{x t}$ and $u_{t x}$, the difference results in zero identically, and the compatibility condition is satisfied as expected.

(2) Consider the case in which $n=3$. In this case,

$$
g^{\prime}\left(z_{x}\right)-g^{\prime}\left(w_{x}\right)=\frac{2 \alpha}{3} v_{x}^{3}+2 \alpha u_{x}^{2} v_{x} .
$$

The coefficient of $v_{x}^{3}$ is a constant, and $\theta(v)$ must satisfy

$$
\frac{\theta^{\prime \prime \prime}(v)}{\theta^{\prime}(v)}=-\frac{2 \alpha}{3} .
$$

Setting $\sigma(v)=\theta^{\prime}(v)$, the order of this equation is reduced by one and a second-order equation results, namely,

$$
\sigma^{\prime \prime}(v)+\frac{2 \alpha}{3} \sigma(v)=0
$$

This equation has trigonometric or hyperbolic function solutions depending on whether $\alpha$ is positive or negative. When $\alpha>0$, the function $\theta(v)$ can be taken from the set

$$
\theta(v)=a \sin (\beta v), \quad \theta(v)=a \cos (\beta v), \quad \beta=\sqrt{\frac{2 \alpha}{3}},
$$

and if $\alpha<0$, we can take $\theta(v)$ from the set

$$
\theta(v)=a \sinh (\beta v), \quad \theta(v)=a \cosh (\beta v), \quad \beta=\sqrt{-\frac{2 \alpha}{3}} .
$$

Here, $a$ is an arbitrary constant. First, $\varphi(v)$ will be determined for the case in which $\theta(v)=a \sin (\beta v)$ so that $u_{x}=a \sin (\beta v)$. To obtain $\varphi(v)$, we compare with the coefficient of $v_{x}$, that is,

$$
-2 \frac{\varphi^{\prime}(v)}{\theta^{\prime}(v)}=2 \alpha a^{2} \sin ^{2}(\beta v) .
$$


This implies that

$$
\varphi^{\prime}(v)=-\alpha a^{3} \beta \sin ^{2}(\beta v) \cos (\beta v)
$$

and hence

$$
\varphi(v)=-\frac{\alpha a^{3}}{3} \sin ^{3}(\beta v)
$$

In this case, the Bäcklund transformation can be written in the form

$$
u_{x}=a \sin (\beta v), \quad u_{t}=-a\left[\beta \cos (\beta v) v_{x x}+\frac{\alpha}{3}\left(v_{x}^{2}+u_{x}^{2}\right) \sin (\beta v)\right], \quad \beta=\sqrt{\frac{2 \alpha}{3}}, \alpha>0 .
$$

This can be repeated for the other functions given in (35) and (36). The results for the hyperbolic sine when $\alpha<0$ are presented as well:

$$
u_{x}=a \sinh (\beta v), \quad u_{t}=-a\left[\beta \cosh (\beta v) v_{x x}+\frac{\alpha}{3}\left(v_{x}^{2}+u_{x}^{2}\right) \sinh (\beta v)\right], \quad \beta=\sqrt{-\frac{2 \alpha}{3}} .
$$

(3) Finally, consider the case in which $n=4$ so that

$$
g^{\prime}\left(z_{x}\right)-g^{\prime}\left(w_{x}\right)=2 \alpha\left(u_{x}^{3} v_{x}+u_{x} v_{x}^{3}\right)
$$

with $u_{x}=\theta(v)(16)$ implies that the function $\theta(v)$ must satisfy the third-order equation

$$
\theta^{\prime \prime \prime}(v)+\alpha\left(\theta(v)^{2}\right)^{\prime}=0 .
$$

Integrating this equation, we obtain

$$
\theta^{\prime \prime}(v)+\alpha \theta(v)^{2}=\kappa,
$$

where $\kappa$ is a constant of integration. Multiplying both sides of this by $\theta^{\prime}$, another integration can be carried out to give the first-order result

$$
\theta^{\prime}(v)^{2}=-\frac{2 \alpha}{3} \theta^{3}+C \theta+C_{1}
$$

The equation can now be written in the form of a quadrature as follows:

$$
\int \frac{d \theta}{\sqrt{C_{1}+C \theta-(2 \alpha / 3) \theta^{3}}}=\epsilon v+C_{2}, \quad \epsilon= \pm 1 .
$$

This will generate a large class of transformations upon integration and then solving for $\theta$ as a function of $v$. The integral can be calculated easily when $C=C_{1}=0$ to give

$$
\theta(v)=-\frac{6}{\alpha} v^{-2}
$$


Then, $\varphi(v)$ is determined by the equation

$$
\varphi^{\prime}(v)=-\alpha \theta^{3}(v) \theta^{\prime}(v)=-\frac{2592}{\alpha^{3} v^{9}},
$$

from which it follows that

$$
\varphi(v)=\frac{6^{4}}{4 \alpha^{3} v^{8}}=\frac{\alpha}{4} u_{x}^{4}
$$

Corresponding to this solution, there exists the following transformation:

$$
u_{x}=\theta(v)=-\frac{6}{\alpha} v^{-2}, \quad u_{t}=-\frac{12}{\alpha} v^{-3} v_{x x}+\frac{18}{\alpha} v^{-4} v_{x}^{2}+\frac{\alpha}{4} u_{x}^{4}
$$

Of course, when $C$ does not vanish, the equation

$$
\theta^{\prime}(v)^{2}+\frac{2 \alpha}{3} \theta(v)^{3}-C \theta(v)-C_{1}=0
$$

has the more complicated solution

$$
\theta(v)=-\mathscr{P}\left(\sqrt{\frac{\alpha}{6}} v+\beta\right)
$$

Here, $\mathscr{P}$ denotes the Weierstrass elliptic function with the invariants $g_{2}=6 \alpha^{-1} \mathrm{C}$ and $g_{3}=6 C_{1} \alpha^{-1}, \beta$ a constant. It therefore follows that the corresponding simple Bäcklund transformation is given by

$$
\begin{gathered}
u_{x}=-\mathscr{P}\left(\sqrt{\frac{\alpha}{6}} v+\beta\right), \\
u_{t}=\sqrt{\frac{\alpha}{6}} \mathscr{P}^{\prime}\left(\sqrt{\frac{\alpha}{6}} v+\beta\right) v_{x x}-\frac{\alpha}{4} \mathscr{P}^{4}\left(\sqrt{\frac{\alpha}{6}}+\beta\right)-\frac{\alpha}{12} \mathscr{P}^{\prime \prime}\left(\sqrt{\frac{\alpha}{6}} v+\beta\right) v_{x}^{2} .
\end{gathered}
$$

At this point, the process cannot be continued for this equation when $n \geq 5$. For example, when $n=5$, the quantity $g^{\prime}\left(z_{x}\right)-g^{\prime}\left(w_{x}\right)$ contains a term proportional to $v_{x}^{5}$, and does not match the form given in (16). However, possible generalizations of Theorem 1 could lead to results for more general forms of the function $g$ which appears in (2).

\section{REFERENCES}

[1] P. Bracken, Symmetry properties of the generalized Korteweg-de Vries equation and some explicit solutions, preprint, 2004.

[2] Some methods for generating solutions to the Korteweg-de Vries equation, Phys. A 335 (2004), no. 1-2, 70-78.

[3] L. Debnath, Nonlinear Partial Differential Equations for Scientists and Engineers, Birkhäuser Boston, Massachusetts, 1997.

[4] Y. S. Kivshar, Intrinsic localized modes as solitons with a compact support, Phys. Rev. E (3) 48 (1993), no. 1, R43-R45.

[5] P. Rosenau and J. M. Hyman, Compactons: solitons with finite wavelength, Phys. Rev. Lett. 70 (1993), no. 5, 564-567.

[6] H. Rund, The Hamilton-Jacobi Theory in the Calculus of Variations. Its Role in Mathematics and Physics, Robert E. Krieger Publishing, New York, 1973. 
[7] _ Variational problems and Bäcklund transformations associated with the sine-Gordon and Korteweg-de Vries equations and their extensions, Bäcklund Transformations, the Inverse Scattering Method, Solitons, and Their Applications (Workshop Contact Transformations, Vanderbilt Univ., Nashville, Tenn, 1974) (R. Miura, ed.), Lecture Notes in Math., vol. 515, Springer, Berlin, 1976, pp. 199-226.

[8] A. J. Sievers and S. Takeno, Intrinsic localized modes in anharmonic crystals, Phys. Rev. Lett. 61 (1988), no. 8, 970-973.

Paul Bracken: Department of Mathematics, University of Texas, Edinburg, TX 78541-2999, USA E-mail address: bracken@panam.edu 


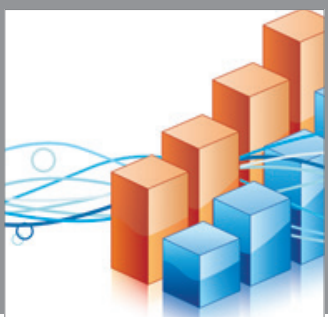

Advances in

Operations Research

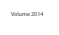

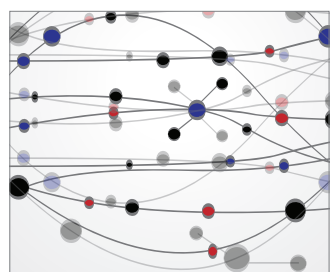

\section{The Scientific} World Journal
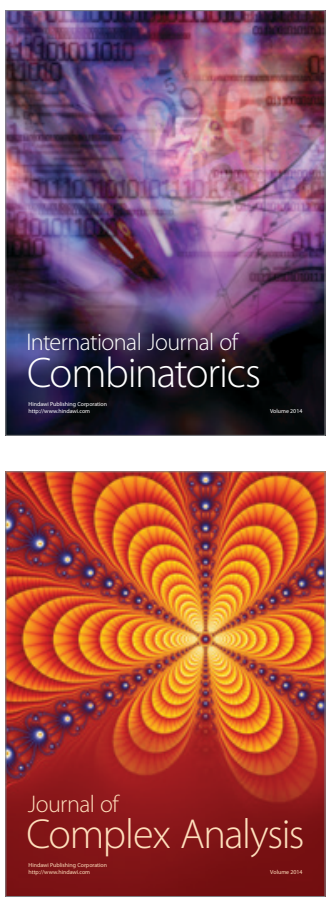

International Journal of

Mathematics and

Mathematical

Sciences
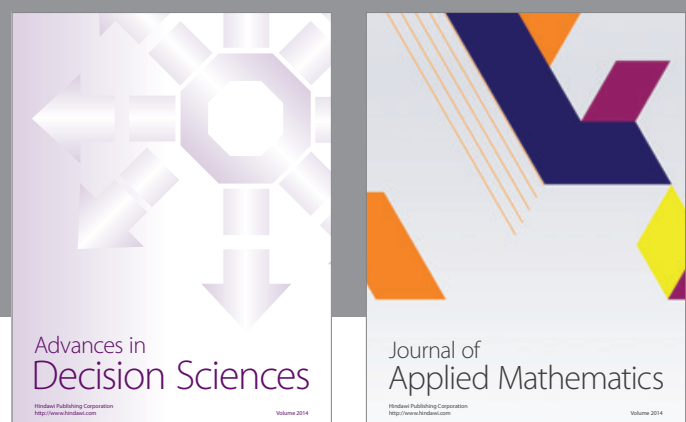

Journal of

Applied Mathematics
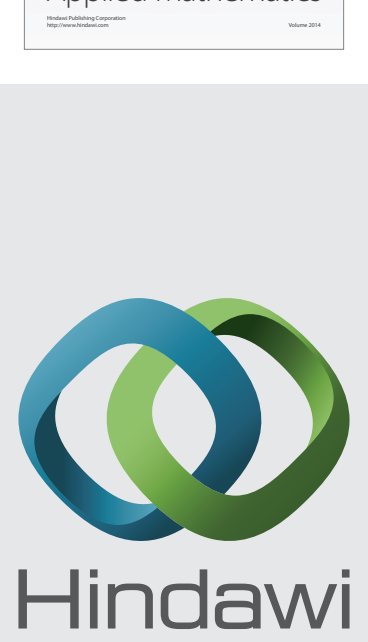

Submit your manuscripts at http://www.hindawi.com
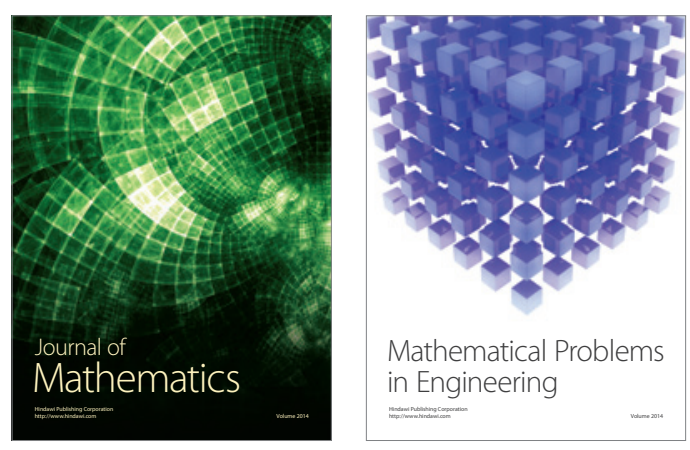

Mathematical Problems in Engineering
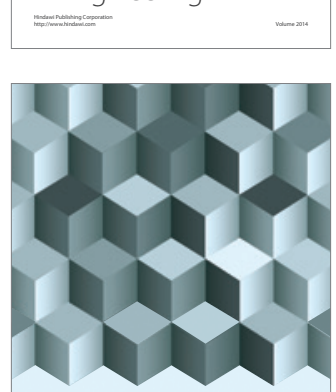

Journal of

Function Spaces
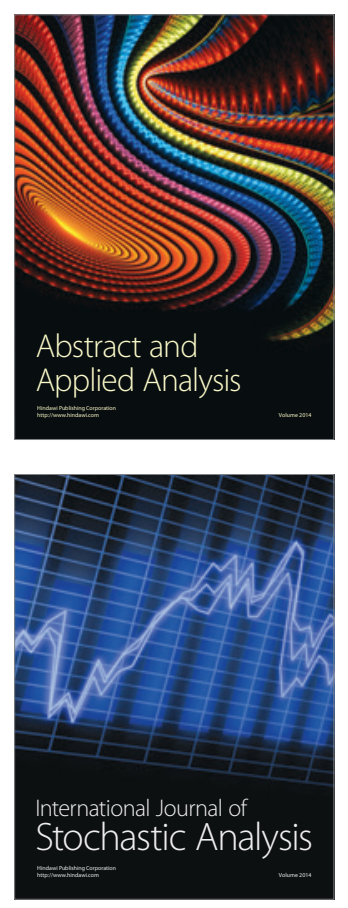

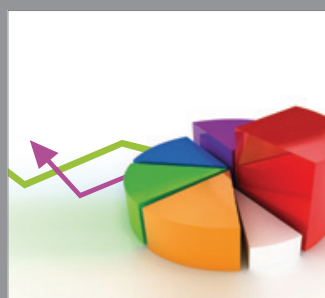

ournal of

Probability and Statistics

Promensencen
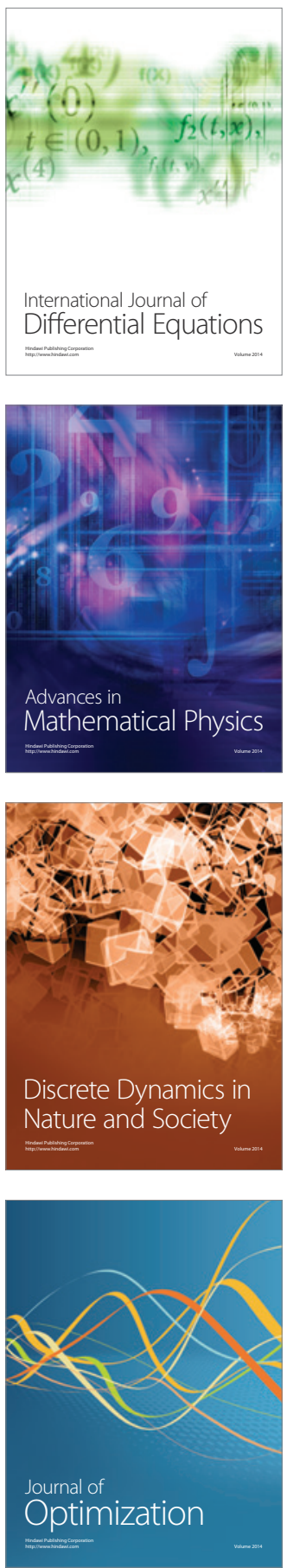